\title{
Chronological Age as Factor Influencing the Dental Implant Osseointegration in the Jaw Bone
}

\author{
Jakub Papež'ㅜ, Tat'jana Dostálová ${ }^{1}$, Karel Chleborád ${ }^{1}$, Pavel Kř́ž ${ }^{1}$, \\ Jakub Strnad ${ }^{2}$ \\ ${ }^{1}$ Department of Stomatology, Second Faculty of Medicine, Charles University \\ and University Hospital Motol, Prague, Czech Republic; \\ ${ }^{2}$ LASAK, Prague, Czech Republic \\ Received October 19, 2017; Accepted March 19, 2018.
}

Key words: Chronological age - Dental implant - Osseointegration - Marginal bone level - Intraoral X-rays

\begin{abstract}
The objectives of this study were to evaluate osseointegration of dental implant in the jaw bone in the young and elderly population and comparing the results to assess indicators and risk factors as age for the success or failure of dental implants. A retrospective study of 107 implants (Impladent, LASAK, Czech Republic) was prepared. The patients at implants surgery were divided in three groups. The patients were followed-up for a 7-year period. We evaluated osseointegration from long term point of view as a change of marginal bone levels close to dental implant. Marginal bone levels were recorded and analysed with regard to different patient- and implant-related factors. An influence of chronological age on change of marginal bone levels during 6-year retrospective study vas evaluated. The study examined 47 patient charts and 107 implants from the Second Faculty of Medicine, Charles University and University Hospital Motol. We proved that young healthy patients with long bridges or Branemarks have the same progression of marginal bone levels changes. The chronological age hasn't therefore direct influence on the osseointegration from long term point of view. But we found that the length of dental suprastrucure-prosthetic construction negatively influences marginal bone changes, though these results weren't
\end{abstract}

This study was supported by project 00064203 (FN MOTOL).

Mailing Address: Prof. Tat’jana Dostálová, MD., DSc., MBA, Department of Stomatology, Second Faculty of Medicine, Charles University and University Hospital Motol,V Úvalu 84, 15006 Prague 5, Czech Republic; Mobile Phone: +420 728970 059; e-mail:Tatjana.Dostalova@fnmotol.cz 
statistically significant. More extensive dental implant suprastrucure undergoes smaller osseointegration. On the other hand the length of dental suprastrucure (prosthetic construction) negatively influences dental osseointegration in both groups of patient.

\section{Introduction}

Osseointegration is seen as the close contact between bone and implant without interposition of non-bone tissue (Albrektsson and Wennerberg, 2004). This concept has been described by Brånemark $(1959,1983)$ as consisting of a highly differentiated tissue making a direct structural and functional connection between ordered, living bone and the surface of a load-carrying implant. Osseointegration of dental implant is influenced on i.e. dental implant surface, age of patient, size, shape and extension of suprastrucure, general health condition, etc.

Various techniques of surfaces treatments have been studied and applied to improve biological surface properties, which favour the mechanism of osseointegration (Wong et al., 1995; Wennerberg and Albrektsson, 2010). This strategy aims at promoting the mechanism of osseointegration with faster and stronger bone formation, to confer better stability during the healing process, thus allowing more rapid loading of the implant (Wennerberg and Albrektsson, 2009; Beutner et al., 2010).

In our article we focused on the age of patient and extend of dental implant suprastrucure as factors influencing bone level changes (osseointegration) during the observed period. The use of dental implants in young patients is not limited, but multidisciplinary treatment planning is directly connected with skeletal maturation. It is evident that jaw growth is important for dental implants insertion. It is known that more and more implants are placed in adolescents, especially after trauma or anodontia.

By adolescent patients we can insert dental implant in the jaw bone only if the growth and skeleton maturation is finished. If implant insertion is planned in a growing child, we must accept the fact that osseointegration forms ankyloses and implants do not follow the spontaneous and continues eruption of the natural dentition (Papez et al., 2015). Similar to ankylosed teeth (Kawanami et al., 1999), the implants remain stationary in the bone and do not follow changes of the alveolar process with continuous eruption of the natural dentition (Oesterle et al., 1993; Cronin et al., 1994). This inability to move with adjacent teeth causes deficiencies in the alveolar bone and surrounding gingival tissues and leads to a discrepancy in the sagittal and transversal dimension, described as infraocclusion or infraposition of the implant (Thilander et al., 1999). Such implants may also disturb the normal development of jawbones. In order not to interfere with the growth of the jawbones, the installation of an implant should generally be postponed on average until after puberty or after the so-called growth spurt of the child (Op Heij et al., 2003). Since changes in the dentoalveolar complex are 
of particular importance for the functional/aesthetic outcome of implants, a study by Iseri and Solow (1996) showed that between the ages of 15 and 25 years the vertical tooth movement can amount to $5 \mathrm{~mm}$ - a distance difficult to span with implants.

The follow-up study of dental implants in the upper adolescent region inserted in adolescent patients, has shown that continuous eruption of the adjacent teeth, even after completed dental and skeletal development, may end up in an infraoccluded implant-supported crown (Thilander et al., 1999). For that reason implant insertion in the anterior tooth area should be postponed until after the completion of the $15^{\text {th }}$ year of age in girls and the $17^{\text {th }}$ year of age in boys, and, therefore, it is necessary to evaluate the upper and lower jaw development. It is known, that the biological indicators of skeletal maturity refer mainly to somatic changes in puberty, thus emphasizing the strict interactions between the development of the craniofacial region and the modifications in other body regions (Baccetti et al., 2005). Individual skeletal maturity can be also assessed by means of several biological indicators: increase in body height; skeletal maturation of the hand and wrist; dental development and eruption; menarche or voice changes; and cervical vertebral maturation (Thilander et al., 1999). For that reason the identification of the pubertal growth spurt has great value in dentistry, mainly in implant insertion area. The effectiveness of a biological indicator of skeletal maturity is directly related to factors such as the ability to detect and predict the growth spurt peak without the need for additional radiation exposure and the high level of agreement between examiners for the definition of the stages (Franchi and Baccetti, 2002). On the opposite site are older patients. Increasing age is strongly associated with the risk of implant failure. Gender, hypertension, coronary artery disease, pulmonary disease, steroid therapy, chemotherapy and not being on hormone replacement therapy for postmenopausal women were not associated with a significant increase in implant failure. Generalized periodontal disease and/or severe periodontal disease negatively influenced the survival probability of the implant (Compton et al., 2017). Smoking, diabetes, head and neck radiation and postmenopausal estrogen therapy are correlated with a significantly increased failure rate (Moy et al., 2005).

\section{Material and Methods}

A retrospective study of 107 implants 47 patients (20 men and 27 women) (Impladent, LASAK, Czech Republic) was prepared.The patients at implants surgery were divided in three groups: group 1 (young patients ranged 18-25 years of age), group 2 (young patients ranged 18-25 years of age), group 3 (older patients ranged 50-60 years of age). The difference between group 1 and 2 was in number of implants and size of suprastrucure. Group 1 are young patients with short suprastrucure that means maximum one bridge connected with two implants or one implant with crown. Group 2 are young patients with extensive suprastrucure, 
more than two implants connected together with one suprastrucure. Group 3 are older patients with extensive suprastrucure, thus more than 2 implants in one suprastrucure. Multidisciplinary therapy by the surgeon, orthodontist, as well as prosthodontist before implant insertion was monitored. The patients were followed-up for a 7-year period, thus time of dental implant installation was minimal 7-year in all implants.

Patient selection

Subject for the study were selected from patients referred to the Department of Stomatology, Second Faculty of Medicine, Charles University and University Hospital Motol. The study was approved by the Ethics Committee of the University Hospital Motol. Informed consent was obtained from all subjects and they were consecutively enrolled in the study according to the predefined inclusion criteria: absence of any local or systemic disease, perfect oral hygiene, non-smokers, without any generalized periodontal disease or severe periodontal disease with sufficient bone height for placing implants. If we inserted the implant in upper jaw we used bone augmentation in all cases.

At the time of selection, patients included in this study showed good general health. After receiving initial therapy including oral hygiene instruction, implantation was performed only after patients had shown good self-performed plaque control. In our study we use two types of implants (implants with sand-blasted surface and implants with bioactive surface) whereas we always compare the same type of implant. If augmentation was necessary (always in upper jaw) during or before surgery we used augmentation material OssaBase ${ }^{\circledR}$-HA (LASAK Ltd.), which is based on synthetic hydroxyapatite.

Table 1 - Statistical analysis of marginal bone level

\begin{tabular}{lrrrrrr}
\hline & \multicolumn{3}{c}{ DIBm } & \multicolumn{3}{c}{ DIBd } \\
\cline { 2 - 7 } & group 1 & group 2 & group 3 & group 1 & group 2 & group 3 \\
\hline Average & 0.155 & 0.046 & -0.135 & 0.060 & -0.006 & -0.037 \\
SD & 0.424 & 0.628 & 1.041 & 0.445 & 0.450 & 0.592 \\
Median & 0.200 & 0.200 & 0.000 & 0.200 & 0.000 & 0.000 \\
Max & 0.700 & 1.300 & 1.300 & 0.600 & 0.900 & 1.400 \\
Min & -1.000 & -1.900 & -5.300 & -1.000 & -1.000 & -2.000 \\
Total number of implants & 20.000 & 35.000 & 52.000 & 20.000 & 35.000 & 52.000 \\
Bone level increase & 16.000 & 26.000 & 32.000 & 15.000 & 21.000 & 32.000 \\
Bone level decrease & 4.000 & 9.000 & 20.000 & 5.000 & 14.000 & 20.000 \\
\hline
\end{tabular}

Group 1 (young patients ranged 18-25 years of age); group 2 (young patients ranged 18-25 years of age with extensive suprastrucure); group 3 (older patients ranged 50-60 years of age). The bone resorption DIB (distance between implant-shoulder to bone-implant contact (Albrektsson et al., 1986) was measured in intraoral X-rays at the both sides of implant (mesial DIBm and distal DIBd). SD - standard deviation 


\section{Treatment procedure}

Following the manufacturer's directions, the fixtures were installed in a randomized order at the edentulous area of each patient. Individual skeletal maturity was checked using skeletal maturation of the hand and wrist by adolescent/young adult patients. In upper jaw we always used bone augmentation. After a healing period of 3 months in the mandible and 6 months in the maxilla, second surgery was performed followed after three weeks by prosthesis delivery. The implants were always inserted in bone level not submerged. During the implant insertion we used the same protocol of implantation. The CAD-CAM technique Zircon Zahn (Prettau ${ }^{\circledR}$ Zirconia, ZirconZahn Gmbhc) and BioCam (LASAK) were used to establish a supra-construction. The patients were recalled every 6 months for through professional plaque control and repeated oral hygiene training. In total, 107 implants (Impladent) were installed (Table 1).

\section{Follow-up parameters}

Clinical examination was conducted every 6 months. An intra-oral digital radiographs (Gendex EXPERT ${ }^{\circledR}$ DC with VistaScan Mini image plate scanner) for each patient were taken from 1 to 7 -year follow-ups. The following clinical variables were recorded: pain from implant regions; implant stability; gingival inflammation; suprastrucure complications; photo and radiographic examination. A periapical digital radiograph (Gendex EXPERT ${ }^{\circledR}$ DC with VistaScan Mini image plate scanner) was taken using the parallel cone technique. We compared bone level in two periods, after fixation of final suprastructure on dental implant and after seven years from implant insertion in the bone. Preventive clinical examination followedup regularly.

\section{Marginal bone-level changes}

During our study we focused on marginal bone-level change thus on resorption of the marginal bone around the implant on the intraoral $X$-rays. The bone resorption DIB (distance between implant-shoulder to bone-implant contact) (Albrektsson et al., 1986) was measured on intraoral X-rays on the both sides of implant (mesial DIBm and distal DIBd) (Figure 1), results were calculated in millimetres with decimal point (e.g. $0.6 \mathrm{~mm}$ ). Calibration was performed with the known length of the fixture. The reference point was the margin of the fixture in cervical part of dental implant. The measurement was done with an accuracy of half a millimetre. Final value DIB was the average DIBm a DIBd, The marginal bone increase for each type of implant was calculated using calibration of radiograph according to length of dental implant (Figure 1). Because we always used the same holder of X-ray sensor and periapical digital radiograph (Gendex EXPERT ${ }^{\circledR}$ DC with VistaScan Mini image plate scanner) with using the parallel cone technique we could set the calibration for all patients and their X-rays. We performed measurements on two sides of dental implants, precisely on mesial and distal aspect of implants; all results 

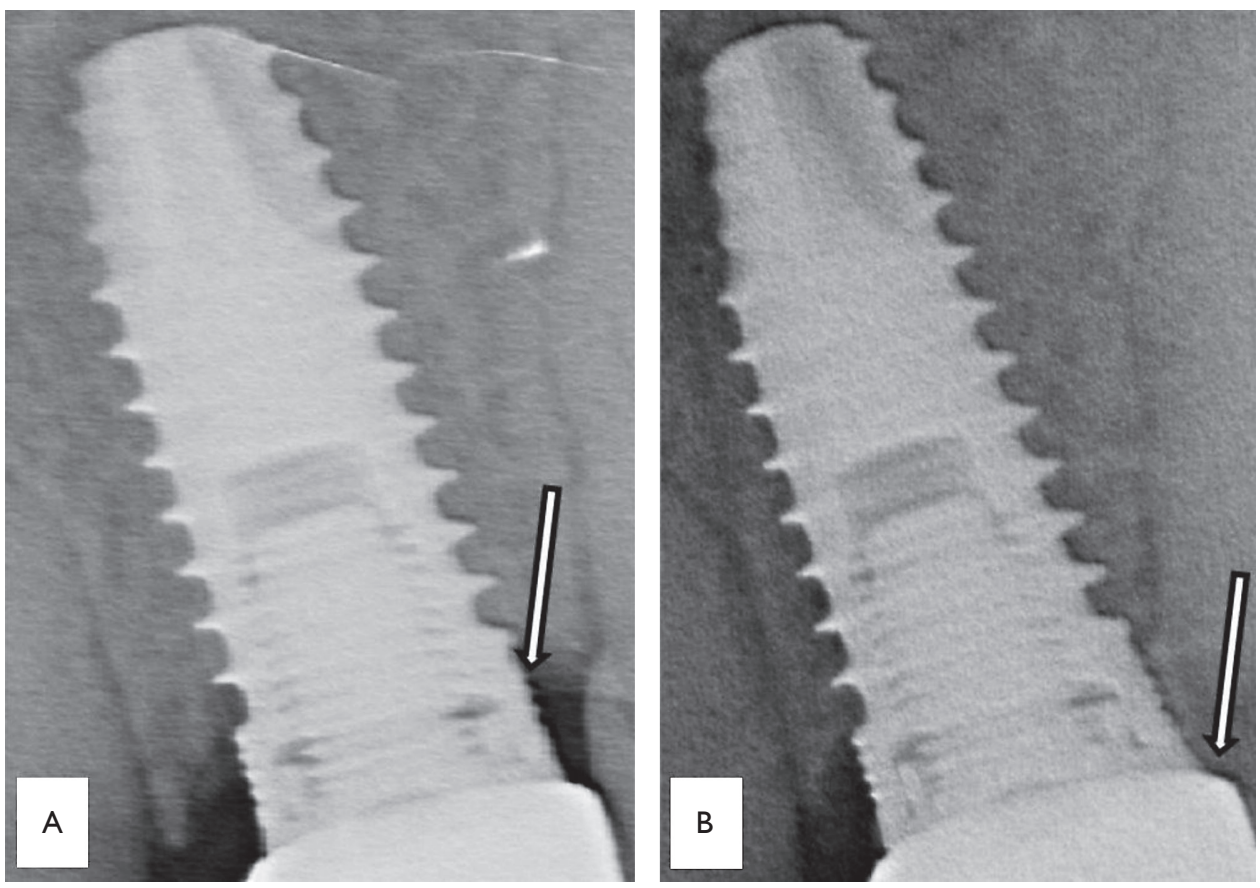

Figure 1 - The marginal bone increase ( $A$ - after therapy; $B-5$ year recall).

were written in table and subsequently statistically evaluated. The parametric tests (two-sample $t$-test, Fisher test) and significance level 0.05 were used for statistical evaluation.

\section{Results}

Clinical examination

No remarkable complications were found during the observation period, no patients suffered from pain, no mobility on implants were detected, and also there were no prosthetic complications.

As is written above, all measured data were statistically evaluated (Tables 1-3).

From the results we concluded, that young and elderly healthy patients with the same number of implants have the same progression of dental implant osseointegration that means that marginal bone level changes were during the retrospective study equal without statistically significant difference, probability less than 0.05 wasn't reached.

On the other hand the length of dental suprastrucure (prosthetic construction) negatively influences dental osseointegration in both groups of patient even if the result isn't statistically significant. More extensive dental implant suprastrucure brings about a higher loss of marginal bone level during the time period. 
Table 2 - Average values which were determined as follows

\begin{tabular}{lllll}
\hline & & DIBm & DIBd & DIB \\
\hline \multirow{2}{*}{ Group 1 } & mean & 0.40 & 0.28 & 0.30 \\
& SD & 0.19 & 0.30 & 0.20 \\
\hline \multirow{2}{*}{ Group 2 } & mean & 0.29 & 0.16 & 0.21 \\
& SD & 0.56 & 0.39 & 0.41 \\
\hline \multirow{2}{*}{ Group 3 } & mean & 0.62 & 0.57 & 0.48 \\
& SD & 0.36 & 0.53 & 0.38 \\
\hline
\end{tabular}

For each person in the group the maximal value was found (Mes., Dist., Mes.+Dist.). From these maximal values averages for each group were calculated. DIB - distance between implant-shoulder to bone-implant contact; $\mathrm{SD}$ - standard deviation

\section{Table 3 - The test of statistical significance was carried out for the differences of average values (Table 2)}

\begin{tabular}{lccc}
\hline & DIBm & DIBd & DIB \\
\hline 1 vs. 3 & 0.141 & 0.189 & 0.242 \\
1 vs. 2 & 0.613 & 0.491 & 0.589 \\
2 vs. 3 & 0.128 & 0.037 & 0.119 \\
$1+2$ vs. 3 & 0.101 & 0.028 & 0.097 \\
\hline
\end{tabular}

Statistically significant difference (probability less than 0.05 ) between the group 2 and $3(0.037)$, and related groups $1+2$ and 3 (0.028). DIB - distance between implant-shoulder to bone-implant contact

\section{Discussion}

Osseointegration of dental implants was defined as the direct, structural, and functional connection between the vital bone and the implant surface under a functional load (Brånemark et al., 1977). Albrektsson et al. (1981) used the new definition that osseointegration is bone tissue formation with no fibrotic layer growth at the bone-implant interface as primarily a biomechanical union. It is a firm, stable, and long-lasting connection between the implant and periimplant bone tissue (Schenk and Buser, 1998).

It is known that congenital partial anodontia and traumatic tooth loss are frequently encountered in pediatric patients. Periodontitis disease or extensive caries are frequently the cause of tooth loss in older patients. Oral rehabilitation in young patient is safe and successful after skeletal and dental maturation. Successful replacement of the lost natural teeth by dental implants is a major advance in clinical dental treatment. The basis of these successful long-term results of endosseous implants depend mainly on the length of the suprastrucure. The radiographic image was the most important source of information for determining the amount of cervical alveolar bone loss or increase around dental implants (Mishra et al., 2013). Relations were evaluated between marginal bone loss around 
implants and the level of the first thread with other systems after 12 months (Kopecká and Šimůnek, 2015). The retrospective study "The success rate of dental implants in elderly" compares the success rate of implants in the edentulous lower jaw in elderly and younger patients. The principal finding of this study was that the long-term success rate of the implants in elderly patients was not lower than that of you with younger patients (Albrektsson et al., 1986). In our article we confirmed that young healthy patient and old healthy patient with the same length of suprastrucure have the same progression of marginal bone loss thus chronological age hasn't direct influence on dental implant osseointegration.

Implants are an alternative to orthodontic space closure, auto transplantation, and conventional prosthetic replacement (Schrotenboer et al., 2008). Implantsupported CAD CAM crowns achieved the best possible long-term result from an aesthetic point of view, and with the least possible distress and suffering for the patient (Behr et al., 2008). Our contribution confirmed the fact that an age is not the decisive factor for implant placement. Only a dental stage indicating fully erupted permanent teeth and skeletal maturation protects dental rehabilitation against infraocclusion of the implant-supported crown.

\section{Conclusion}

Osseointegration is necessary for successful dental implant insertion in the jaw bone and ensures the stability of dental implant from long term point of view. As we know from our results when we compare healthy young and healthy old patients the marginal bone loss in the mentioned groups does not show statistically significant differences.

The length of dental suprastrucure-prosthetic construction negatively influences dental osseointegration; more precisely it can progressively change the alveolar bone around the implant during the observed time period. More extensive dental implant suprastrucure has a negative influence on the marginal bone levels.

\section{References}

Albrektsson, T., Wennerberg, A. (2004) Oral implant surfaces: Part 1 - Review focusing on topographic and chemical properties of different surfaces and in vivo responses to them. Int. J. Prosthodont. 17, 536-543.

Albrektsson, T., Brånemark, P. I., Hansson, H. A., Lindström, J. (1981) Osseointegrated titanium implants. Requirements for ensuring a long-lasting, direct bone-to-implant anchorage in man. Acta Orthop. Scand. 52, 155-170.

Albrektsson, T., Zarb, G., Worthington, P., Eriksson, R. A. (1986) The long-term efficacy of currently used dental implants: A review and proposed criteria for success. Int. J. Oral Maxillofac. Implants 1, 11-25.

Baccetti, T., Franchi, L., McNamara, J. A. (2005) The cervical vertebral maturation (CVM) method for the assessment of optimal treatment timing in dentofacial orthopedics. Semin. Orthod. 11, 119-129.

Behr, M., Driemel, O., Mertins, V., Gerlach, T., Kolbeck, C., Rohr, N., Reichert, T. E., Handel, G. (2008) Concepts for the treatment of adolescent patients with missing permanent teeth. Oral Maxillofac. Surg. 12, 49-60.

Beutner, R., Michael, J., Schwenzer, B., Scharnweber, D. (2010) Biological nano-functionalization of titaniumbased biomaterial surfaces: a flexible toolbox. J. R. Soc. Interface 7, 93-105.

Papež J.; Dostálová T.; Chleborád K.; Kř́ž P.; Strnad J. 
Brånemark, P. I. (1959) Vital microscopy of bone marrow in rabbit. Scand. J. Clin. Lab. Invest. 11, 1-82. Brånemark, P. I. (1983) Osseointegration and its experimental studies. J. Prosthet. Dent. 50, 399-410.

Brånemark, P. I., Hansson, B. O., Adell, R., Breine, U., Lindström, J., Hallén, O. (1977) Osseointegrated implants in the treatment of the edentulous jaw. Experience from a 10-year period. Scand. J. Plast. Reconstr. Surg. 16, 1-132.

Compton, S. M., Clark, D., Chan, S., Kuc, I., Wubie, B. A., Levin, L. (2017) Dental implants in the elderly population: a long-term follow-up. Int. J. Oral Maxillofac. Implants 32, 164-170.

Cronin, R. J. Jr., Oesterle, L. J., Renly, D. M. (1994) Mandibular implants and the growing patient. Int. J. Oral Maxillofac. Implants 9, 55-62.

Franchi, L., Baccetti, T. (2002) The use of maturational indices for the identification of optimal treatment timing in dentofacial orthopedics. In: Treatment Timing: Orthodontics in Four Dimensions. Craniofacial Growth Series, Vol. 39. McNamara, J. A. Jr., Kelly, K. A., eds., pp. 151-170, Center for Human Growth and Development, University of Michigan, Ann Arbor.

Iseri, H., Solow, B. (1996) Continued eruption of maxillary incisors and first molars in girls from 9 to 25 years, studies by the implant method. Eur. J. Orthod. 18, 246-256.

Kawanami, M., Andreasen, J. O., Borum, M. K., Schou, S., Hjorting-Hansen, E., Kato, H. (1999) Infraposition of ankylosed permanent maxillary incisors after replantation related to age and sex. Endod. Dent. Traumatol. 15, 50-56.

Kopecká, D., Šimůnek, A. (2015) Úspěšnost dentálních implantací ve vyšším věku (The success rate of dental implants in elderly). Česká Stomatologie 115,13-17. (in Czech)

Mishra, S. K., Chowdhary, N., Chowdhary, R. (2013) Dental implants in growing children. J. Indian Soc. Pedod. Prev. Dent. 31, 3-9.

Moy, P. K., Medina, D., Shetty, V., Aghaloo, T. L. (2005) Dental implant failure rates and associated risk factors. Int. J. Oral Maxillofac. Implants 20, 569-577.

Oesterle, L. J., Cronin, R. J. Jr., Renly, D. M. (1993) Maxillary implants and the growing patient. Int. J. Oral Maxillofac. Implants 8, 377-387.

Op Heij, D. G., Opdebeeck, H., van Steenberghe, D., Quirynen, M. (2003) Age as compromising factor for implant insertion. Periodontol 2000 33, 172-184.

Papez, J., Dostalova, T., Kriz, P., Chleborad, K., Polaskova, A., Feberova, J. (2015) Dental implants and skeleton maturation as factors influenced implant insertion by adolescent patients with missing permanent teeth: 5-year prospective study. IOSR-JDMS 14, 43-48.

Schenk, R. K., Buser, D. (1998) Osseointegration: a reality. Periodontol 2000 17, 22-35.

Schrotenboer, J., Tsao, Y., Kinariwala, V., Wang, H. (2008) Effect of microthreads and platform switching on crestal bone stress levels: a finite element analysis. J. Periodontol. 79, 2166-2217.

Thilander, B., Odman, J., Jemt, T. (1999) Single implants in the upper incisor region and their relationship to the adjacent teeth. An 8-year follow-up study. Clin. Oral Implants Res. 10, 346-355.

Wennerberg, A., Albrektsson, T. (2009) Effects of titanium surface topography on bone integration: a systemic review. Clin. Oral Implants Res. 20,172-184 (Suppl. 4).

Wennerberg, A., Albrektsson, T. (2010) On implant surfaces: a review of current knowledge and opinions. Int. J. Oral Maxillofac. Implants 25, 63-75.

Wong, M., Eulenberger, J., Schenk, R., Hunziker, E. (1995) Effect of surface topology on the osseointegration of implant materials in trabecular bone. J. Biomed. Mater. Res. 29, 1567-1575. 